\title{
Glucose detection in a highly scattering medium with diffuse photon-pair density wave
}

\author{
Li-Ping $\mathrm{Yu}^{*, \dagger}$, Jheng-Syong $\mathrm{Wu}^{\dagger, \dagger}$, Sheng-Yi Chang, ${ }^{\S}$, and Chien $\mathrm{Chou}^{*, \dagger}, \|$ \\ *Biomedical Engineering Research Center, Chang Gung University \\ Taoyuan 333, Taiwan \\ ${ }^{\dagger}$ Graduate Institute of Electro-Optical Engineering, Chang Gung University \\ Taoyuan 333, Taiwan \\ *Institute of Chemistry, Academic Sinica, Nankang, Taipei 115, Taiwan \\ ${ }^{\S}$ Ph.D Program of Biomedical Engineering, Chang Gung University \\ Taoyuan 333, Taiwan \\ "Department of Mechanics, Ming Chi University of Technology \\ New Taipei 243, Taiwan \\ "cchou01@gmail.com
}

Received 28 September 2015

Accepted 2 February 2016

Published 18 March 2016

\begin{abstract}
We propose a novel optical method for glucose measurement based on diffuse photon-pair density wave (DPPDW) in a multiple scattering medium (MSM) where the light scattering of photonpair is induced by refractive index mismatch between scatters and phantom solution. Experimentally, the DPPDW propagates in MSM via a two-frequency laser (TFL) beam wherein highly correlated pairs of linear polarized photons are generated. The reduced scattering coefficient $\mu_{2 s}^{\prime}$ and absorption coefficient $\mu_{2 a}$ of DPPDW are measured simultaneously in terms of the amplitude and phase measurements of the detected heterodyne signal under arrangement at different distances between the source and detection fibers in MSM. The results show that the sensitivity of glucose detection via glucose-induced change of reduced scattering coefficient $\left(\delta \mu_{2 s}^{\prime}\right)$ is $0.049 \%$ $\mathrm{mM}^{-1}$ in a $1 \%$ intralipid solution. In addition, the linear range of $\delta \mu_{2 s}^{\prime}$ vs glucose concentration implies that this DPPDW method can be used to monitor glucose concentration continuously and noninvasively subcutaneously.
\end{abstract}

Keywords: Diffuse photon-pair density wave; glucose; multiple scattering; polarization gating; spatial coherence gating.

\|Corresponding author.

This is an Open Access article published by World Scientific Publishing Company. It is distributed under the terms of the Creative Commons Attribution 4.0 (CC-BY) License. Further distribution of this work is permitted, provided the original work is properly cited. 


\section{Introduction}

Conventional approaches to measuring glucose in a nonscattering medium uses optical rotation of linear polarized light and detection of the amplitude or phase change of the emergent polarized laser beam vs the incident beam. ${ }^{1,2}$ For glucose measurements in a multiple scattering medium (MSM), larger error results from optical rotation angle measurements. This is due to multiple scattering that induces multiple optical path lengths of laser beam in the media. Moreover, the depolarization of the linear or circular polarized laser beam in MSM is also sensitive to optical rotation and angle measurements. ${ }^{3}$ As a result, the measurement of glucose concentration in turbid media becomes difficult via the optical rotation method. ${ }^{4}$ Alternatively, glucose in MSM can reduce the light scattering due to a reduced refractive index mismatch between scatters and the surrounding medium.

Hence, Maier et al. ${ }^{5}$ and Kohl et al. ${ }^{6}$ proposed a method that focuses on reduced scattering coefficient $\mu_{s}^{\prime}$ measurements via diffuse photons while the absorption coefficient $\mu_{a}$ was ignored due to the properties of $\mu_{s}^{\prime}>>\mu_{a}$ in the near infrared (NIR) spectral region. Bruulsema et al. ${ }^{7}$ also proposed to measure glucose concentrations noninvasively in terms of the $\mu_{s}^{\prime}$ of the tissue via eight detection fibers that were located at different distances from the source fiber using a multichannel CCD. The $\mu_{s}^{\prime}$ of the human tissue is dependent on the refractive index mismatch between the extracellular fluid (ECF) and cellular membrane. A higher glucose concentration increases the refractive index of ECF and decreases mismatch of refractive indices. Thus, $\mu_{s}^{\prime}$ decreases, too. In fact, the absorption of tissue is also involved during $\mu_{s}^{\prime}$ measurements, and both $\mu_{s}^{\prime}$ and $\mu_{a}$ are refractive-index dependent and are expressed by $n \mu_{s}^{\prime}$ and $\mu_{a} / n$, respectively, according to linear diffuse photon transport theory where $n$ is the refractive index of ECF. According to the BeerLambert law, total attenuation coefficient of tissue is considered during measurement. Hence, both $\mu_{s}^{\prime}$ and $\mu_{a}$ are included in the detected signal.

Recently, optical coherence tomography (OCT) has been proposed to measure glucose concentration in the dermis that is focused on ballistic photons (not scattered photons) rather than scattered photons via heterodyne detection ${ }^{8,9}$ where the total attenuated amplitude of heterodyne signal at different depths is measured. Thus, the slope of OCT signal vs $\mu_{s}^{\prime}$ as a function of glucose concentration was demonstrated. However, the accuracy of this method is highly dependent on tissue heterogeneity and the absorption coefficient. Thus, a NIR laser was used to remove the absorption in the dermis because of $\mu_{s}^{\prime}>>\mu_{a}$ in the NIR spectral region. When $\mu_{a}$ is comparable with $\mu_{s}^{\prime}$, the absorption effect becomes non negligible. Besides, a high frequency intensity-modulated laser diode is introduced to generate a diffuse photon density wave (DPDW) in MSM whereas the diffuse photons in MSM follow the diffusion equation. Both $\mu_{s}^{\prime}$ and $\mu_{a}$ can be measured simultaneously via the amplitude and phase of the emerging intensity-modulated laser beam vs the input beam. The glucose concentration is then obtained in terms of $\mu_{s}^{\prime}$ only at higher accuracy. The absorption effect is obviously removed. Using this approach, the $\mu_{a}$-dependent physiological parameters such as oxygenatedhemoglobin $\left(\mathrm{HbO}_{2}\right)$ and hemoglobin saturation by oxygen $\left(\mathrm{StO}_{2}\right)$ can be measured independently via multiple wavelengths. Experimentally, the result of increasing glucose concentration induces decreasing $\mu_{s}^{\prime}$ in selected human tissue. This reduces the scattering coefficient $\delta \mu_{s}^{\prime} \sim 0.3 \% \mathrm{mM}^{-1}$ that was predicted theoretically by Kohl et al. ${ }^{6}$ Meanwhile, the $\delta \mu_{s}^{\prime}$ was larger at $0.34 \% \mathrm{mM}^{-1}$ and $0.6 \% \mathrm{mM}^{-1}$ in the abdomen and thigh of human subjects, respectively. ${ }^{5,7}$ Nevertheless, high intensity modulation frequency $(>100 \mathrm{MHz})$ is required in the DPDW method, and noise from radio frequency is then generated during measurements. ${ }^{5-8}$ This limits glucose sensitivity in the dermis particularly for blood glucose detection related to diabetes.

In this study, we describe a novel method for noninvasive glucose concentration detection. This is based on our previously proposed diffuse photonpair density wave (DPPDW). The glucose concentration can be measured in terms of reduced scattering coefficients $\mu_{2 s}^{\prime}$ and absorption coefficients $\mu_{2 a}$ that were measured by photon pairs. ${ }^{10,11}$ In contrast to DPDW, the DPPDW in MSM uses lower amplitude modulation frequency $(20 \mathrm{MHz})$ via heterodyne interference and highly correlated polarized photon pairs (PPPs). A two-frequency $\mathrm{He}-\mathrm{Ne}$ laser produces DPPDW in turbid media, and the amplitude and phase of the heterodyne signal are detected simultaneously. During measurements, two different gatings - the polarization gating and spatial coherence gating - are functioned together concurrently. Thus, $\mu_{2 s}^{\prime}$ and $\mu_{2 a}$ of 
the tested turbid media such as intralipid solution are obtained simultaneously. The DPPDW-based method shows high signal-to-noise ratio (SNR) of the detected heterodyne signal. This simultaneously increases sensitivity of $\mu_{2 s}^{\prime}$ and $\mu_{2 a}$ measurements.

Finally, glucose concentration detection via $\mu_{2 s}^{\prime}$ only (without absorption effect) at high detection sensitivity is performed. Moreover, the physiological parameters such as $\mathrm{HbO}_{2}$ and $\mathrm{StO}_{2}$ in terms of $\mu_{2 a}$ without scattering effect are applicable via multiple wavelengths. ${ }^{12-15}$ In this experiment, the glucose concentration measurements were done in $1 \%$ intralipid solution at a detection sensitivity of $\delta \mu_{2 s}^{\prime}=0.049 \% \mathrm{mM}^{-1}$ with an overall linear range of 0-35 mM. This indicates that the DPPDW-based method offers higher sensitivity for $\mu_{2 s}^{\prime}$ than conventional methods using diffuse photons. To further improve the detection sensitivity of this proposed method into the $2 \mathrm{mM}$ physiological range for diabetes is also discussed.

\section{Working Principle}

According to DPPDW theory, ${ }^{10,11}$ the heterodyne signal in an infinite, homogenous turbid medium is expressed as:

$$
I(r, t)=\gamma A_{1} A_{2} \frac{\exp \left(-k_{2 r} r\right)}{2 \pi D r} \cos (\Delta \omega t-\Delta \Theta),
$$

where $A_{1}$ and $A_{2}$ are the amplitudes of the paired photons, respectively. The $\Delta \omega$ is the beat frequency of the heterodyne signal, $D$ is defined as $\left[3\left(\mu_{2 a}+\mu_{2 s}^{\prime}\right)\right]^{-1}$ where $\mu_{2 a}$ and $\mu_{2 s}^{\prime}$ are the PPPs absorption coefficient $\left(\mathrm{cm}^{-1}\right)$ and reduced scattering coefficient $\left(\mathrm{cm}^{-1}\right)$, respectively. Term $\gamma$ is the heterodyne efficiency determined by the spatial coherence and degree of polarization of the PPP. ${ }^{16}$ As a result, moderately scattering PPP is selected via spatial coherence gating and polarization gating. ${ }^{10,11}$ The real part of the wavenumber $k_{2 r}$ of DPPDW is given by

$$
k_{2 r}=\left(\mu_{2 a} / D\right)^{1 / 2} .
$$

The phase difference $\Delta \Theta$ between the pair of photons after propagating a distance is given by

$$
\Delta \Theta=\frac{\Delta \omega}{v} \tilde{r}=\frac{\Delta \omega}{v}\left(\frac{3 \mu_{2 s}^{\prime}}{4 \mu_{2 a}}\right)^{1 / 2} r,
$$

where $\tilde{r}$ is the average optical path in the turbid medium, which is the product of $\left(3 \mu_{2 s}^{\prime} / 4 \mu_{2 a}\right)^{1 / 2}$ and the geometrical distance $r$ between the source fiber and the detector fibers; $v$ is the speed of light in a turbid medium. The imaginary part of wave number $k_{2 i}$ is expressed by

$$
k_{2 i} \equiv \frac{\Delta \omega}{v}\left(\frac{3 \mu_{2 s}^{\prime}}{4 \mu_{2 a}}\right)^{1 / 2} .
$$

From Eqs. (1), (3) and (4), $I(r)$ and $\Theta$ are linearly related to $r$ and expressed by

$$
\begin{aligned}
\operatorname{In}[r \mathrm{I}(r)] & =-k_{2 r} r+\operatorname{In}\left(\mathrm{I}_{0}\right), \\
\Theta & =k_{2 i} r+\theta,
\end{aligned}
$$

where $I_{0}$ and $\theta$ are the initial amplitude and phase of DPPDW. The values of $k_{2 r}$ and $k_{2 i}$ can be determined by measuring the amplitude and phase of DPPDW at multiple distances from the source fiber. Thus, the optical properties of $\mu_{2 a}$ and $\mu_{2 s}^{\prime}$ are presented by

$$
\begin{aligned}
& \mu_{2 s}^{\prime}=2 v k_{2 r} k_{2 i} /(3 \Delta \omega), \\
& \mu_{2 a}=\frac{\Delta \omega}{2 v}\left(\frac{k_{2 r}}{k_{2 i}}\right) .
\end{aligned}
$$

\section{Experimental Setup}

The experimental setup for measurement of glucose in $1 \%$ intralipid solution is shown in Fig. 1. A twofrequency He-Ne laser source (Zygo 7702 laser head, USA) outputs two orthogonally linear polarized light waves ( $\mathrm{P}$ and $\mathrm{S}$ waves) with a frequency difference at $20 \mathrm{MHz}$, generated by two acoustic modulators. The center wavelength of the laser is $632.8 \mathrm{~nm}$. Along the optical path, a polarizer at a $45^{\circ}$ azimuth angle to both $P$ and $S$ waves is mounted. It generates a pair of highly correlated

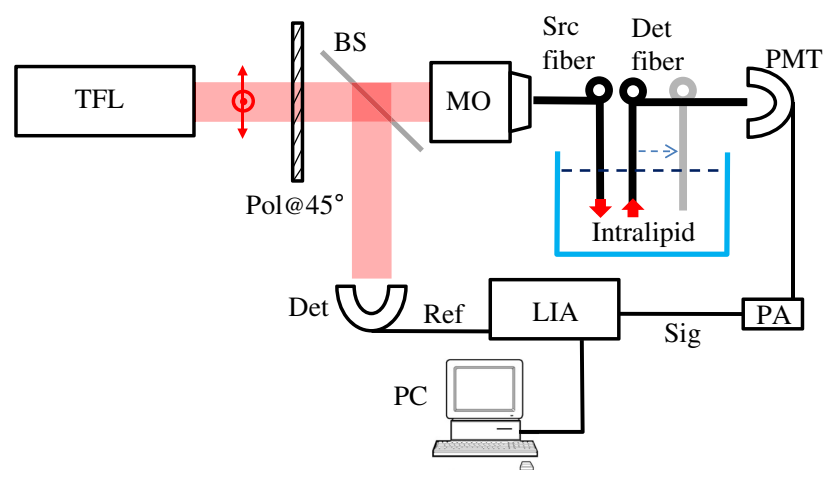

Fig. 1. Experimental setup of DPPDW: TFL, two-frequency laser; Pol, polarizer; BS, beam-splitter; MO, microscope objective; PMT, photomultiplier tube; Det, photodetector; PA, preamplifier; and LIA, lock-in amplifier. 
parallel linear polarized light beam called the PPP beam.

Polarized photon-pair laser beams were divided into a reference beam and a signal beam. The reference beam produces a $20 \mathrm{MHz}$ beat signal with a photodetector that is connected to the reference channel of a lock-in amplifier (LIA) (Stanford Research SR844, USA). The signal beam is guided into $1 \%$ intralipid solution via a source fiber of $1 \mathrm{~mm}$ core diameter and a $\mathrm{NA}=0.37$. The emitted power from the source fiber is $0.2 \mathrm{~mW}$. Meanwhile, the second fiber (detection fiber) is controlled by a motorized stage and is scanned in a reflectance geometry to collect the diffuse PPP from the source fiber at given distances. There are six positions of the detection fiber from the source fiber under arrangement of 1.5 to $3 \mathrm{~cm}$ of source-to-detector distance. This measures the optical properties of the $1 \%$ intralipid solution. Finally, diffuse PPP is detected with a photomultiplier tube (PMT) associated with a preamplifier (PA) that converts the current to voltage of the detected signal. The amplitude and phase of the heterodyne signal are measured with a LIA.

In the experiment, a $1 \%$ intralipid solution was made by 10 -fold dilution from commercially available $10 \%$ intralipid solution (Fresenius Kabi AB, Sweden). The size was $20 \times 20 \times 15 \mathrm{~cm}$. It can be treated as an infinite geometry for diffuse photon detection. The reduced scattering coefficient and absorption coefficient of $1 \%$ intralipid solution are $\sim 10 \mathrm{~cm}^{-1}$ and $\sim 0.01 \mathrm{~cm}^{-1}$, respectively. ${ }^{15,17}$ Glucose concentration was increased step by step in $1 \%$ intralipid solution with an increase of $5 \mathrm{mM}$ from $0-35 \mathrm{mM}$. This is the range of interest for diabetic patients. Smaller increases at $2 \mathrm{mM}$ or $3 \mathrm{mM}$ from $0-10 \mathrm{mM}$ or $0-24 \mathrm{mM}$ were applied to find the detection limits. During measurements, a uniform distribution of glucose concentration in intralipid solution is required while the temperature was kept at $25^{\circ} \mathrm{C}$. Higher absorption coefficients are considered if high output power of TFL is introduced.

\section{Experimental Results}

Figure 2 shows the experimental results of the measurements of $\mu_{2 s}^{\prime}$ as a function of glucose concentration in a $1 \%$ intralipid solution with three different increments and ranges of glucose concentrations. The increase per step of glucose measurements are (a) $5 \mathrm{mM}$ in the concentration range of $0-35 \mathrm{mM}$, (b) $3 \mathrm{mM}$ in the range of $0-24 \mathrm{mM}$, and (c) $2 \mathrm{mM}$ in the range of $0-10 \mathrm{mM}$ (Figs. 2(a)-2(c)), respectively. The vertical axis in Fig. 2 is defined by the change of $\mu_{2 s}^{\prime}$ (i.e., $\delta \mu_{2 s}^{\prime}$ ) relative to the control that is at $0 \mathrm{mM}$ glucose concentration in a $1 \%$ intralipid solution. Figure 2(d) presents all measurements presented in Figs. 2(a)-2(c).

The range of glucose concentration in Figs. 2(a) and 2(b) correspond to diabetic patients while the glucose range $(0-10 \mathrm{mM})$ in Fig. $2(\mathrm{c})$ is for healthy controls. Linear regression was applied in Fig. 2 to determine the sensitivity of $\delta \mu_{2 s}^{\prime}$ vs glucose concentration. They are (a) $0.045 \% \mathrm{mM}^{-1}$, (b) $0.05 \%$ $\mathrm{mM}^{-1}$, and (c) $0.043 \% \mathrm{mM}^{-1}$ in Figs. 2(a)-2(c), respectively. Finally, the sensitivity $\delta \mu_{2 s}^{\prime}$ of the overall measured range shown in Fig. $2(\mathrm{~d})$ is $0.049 \%$ $\mathrm{mM}^{-1}$. The correlation coefficients $\mathrm{R}$ at Fig. 2(a) $0.96(p<0.001)$, Fig. 2(b) $0.91(p<0.001)$, Fig. 2(c) $0.67(p>0.1)$, and Fig. 2(d) $0.88(p<0.001)$ are shown in Figs. 2(a)-2(d), respectively. The reduced scattering coefficient $\mu_{2 s}^{\prime} \sim 14.5 \mathrm{~cm}^{-1}$ was obtained in this experiment and is consistent with our previous experimental result ${ }^{10}$ of $\mu_{2 s}^{\prime} \sim 19 \mathrm{~cm}^{-1}$ in a $1.5 \%$ intralipid solution. Most of the results show strong correlation $(R>0.84)$ and significance $(p<0.001)$ of glucose concentration on $\delta \mu_{2 s}^{\prime}$. The error bar in each measurement is the standard deviation over 10 independent measurements. This is $0.1 \%$ on average for all measurements. This indicates the repeatability of these measurements.

\section{Discussion and Conclusions}

In this study, we propose and demonstrate a novel method of using a two-frequency linear polarized laser beam following the theory of DPPDW in turbid media for glucose concentration measurements. The glucose concentration in $1 \%$ intralipid solution was measured whereas the detection sensitivity of $\delta \mu_{2 s}^{\prime}=0.049 \% \mathrm{mM}^{-1}$ was reached. This is consistent with the theoretical prediction of $0.05 \% \mathrm{mM}^{-1}$ by Kohl et al. ${ }^{6}$ Meanwhile, the experimental result agrees with $0.036 \% \mathrm{mM}^{-1}$ in $1.5 \%$ Liposyn-glucose suspension by Maier et al..$^{5}$ using a NIR light source. At an accuracy of $0.1 \%$ of $\delta \mu_{2 s}^{\prime}$ measurements, the calculated detection limit of glucose concentration in this method is $4 \mathrm{mM}$ according to the definition by The International Union of Pure and Applied Chemistry, ${ }^{18}$ when we focused on glucose concentration measurements in the range of $0-35 \mathrm{mM}$ (Fig. 2(a)) and $0-24 \mathrm{mM}$ 


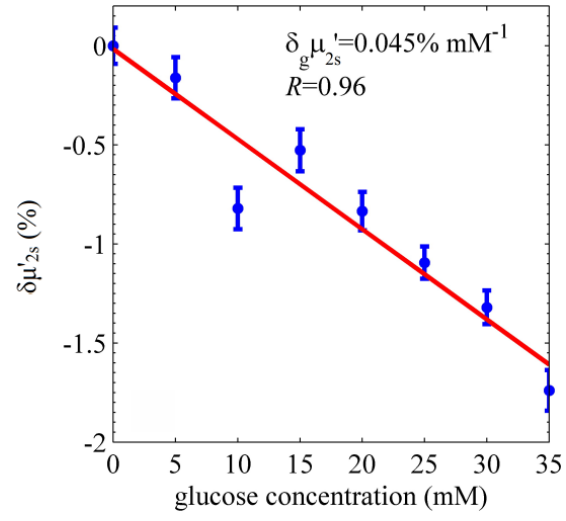

(a)

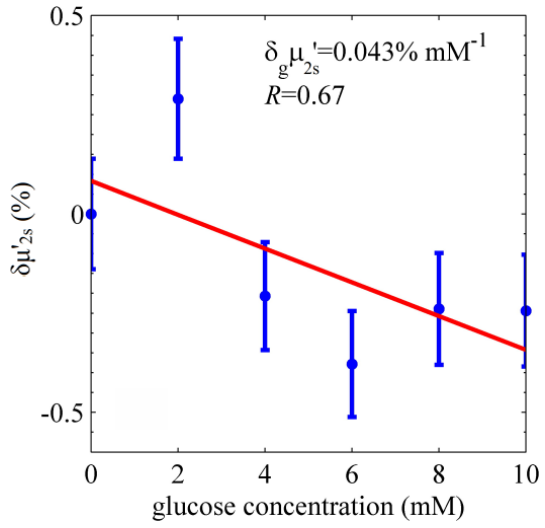

(c)

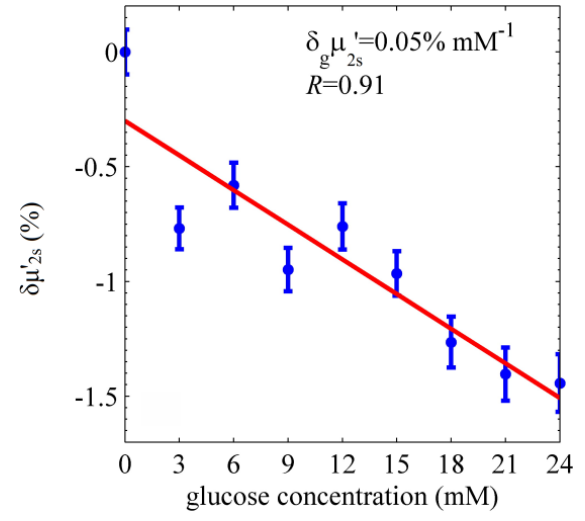

(b)

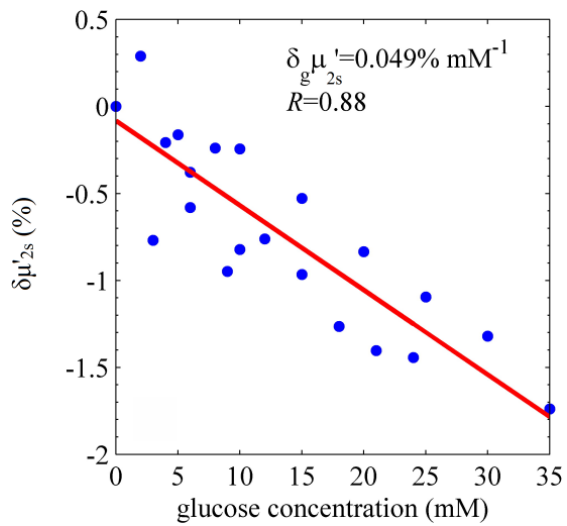

(d)

Fig. 2. The experimental results of the fractional changes (\%) of $\mu_{2 s}^{\prime}$ in $1 \%$ intralipid suspension via variation of glucose concentration. The increase per step of glucose measurements are (a) $5 \mathrm{mM}$ in the concentration range of $0-35 \mathrm{mM}$, (b) $3 \mathrm{mM}$ in the range of $0-24 \mathrm{mM}$, and (c) $2 \mathrm{mM}$ in the range of $0-10 \mathrm{mM}$ as shown in Figs. 2(a)-2(c), respectively. The error bar represents the standard deviation of 10 measurements. Figure 2(d) shows all measurements presented in Figs. 2(a)-2(c).

(Fig. 2(b)). This result was further verified in the measurement shown in Fig. 2(b) where the detection is an increase of $3 \mathrm{mM}$ glucose with high confidence $(R=0.91, p<0.001)$. However, when the resolution of glucose concentration was set at $2 \mathrm{mM}$, the measured result did not show reliable measurements under $R=0.67, p>0.1$ as shown in Fig. 2(c). This might be because of a nonuniform distribution of glucose in the sample. The DPPDW method in this study shows better detection sensitivity due to heterodyne detection via polarization and spatial coherence gatings. ${ }^{5,6,9}$ Because of a relatively larger variation in the range $0-10 \mathrm{mM}$ of glucose concentration as shown in Fig. 2(c), a TFL with higher beat frequency and larger output power is suggested in this setup for better performance according to our previous discussion using DPPDW. ${ }^{10,11}$ Generally, the absorption effect can be ignored in most glucose detection methods via NIR light. ${ }^{5,6,9}$ However, for high absorption coefficient of MSM such as human tissue, the absorption effect cannot be ignored. Hence, the ability of simultaneous measurements of $\mu_{2 a}$ and $\mu_{2 s}^{\prime}$ becomes critical to reach high sensitivity glucose concentration. ${ }^{7,19}$ As a result, this proposed method based on DPPDW can measure the reduced scattering coefficient and absorption coefficient simultaneously at higher sensitivity using a moderate beat frequency of heterodyne signal. This becomes one of the advantages of this setup vs conventional methods of glucose monitoring noninvasively for diabetes.

To conjugate properties of DPPDW with optical heterodyne detection wherein the polarization gating, spatial coherence gating are functioned together simultaneously and a perfect sinusoidal modulation signal is generated in this DPPDW-based method due to optical heterodyne interference. Hence, high SNR in the heterodyne signal results in high sensitivity for $\mu_{2 s}^{\prime}$ and $\mu_{2 a}$ 
measurements. In addition, a semi-infinite geometry ${ }^{20}$ or slab geometry ${ }^{21}$ can be applied in DPPDW method because of the diffusion equation of PPP. If multiple wavelengths of light are used in this setup, the absorption coefficient dependent on physiological parameters such as hemoglobin oxygen saturation is also measured. However, because temperature, osmolytes, cell volume changes, and humidity affect tissue-scattering properties, the quantitative measurement of glucose concentration in human tissue requires carefully calibration. Therefore, applying this method to homecare glucose monitoring is motivated due to its continuous glucose monitoring ability particularly in type I diabetes because of daily blood glucose assays.

\section{Acknowledgment}

The research was supported by Ministry of Science and Technology (MOST) of Taiwan.

\section{References}

1. G. R. Fowels, Introduction to Modern Optics, 2nd Edition, Dover Publications, New York (1989).

2. C. Chou, C. Y. Han, W. C. Kuo, Y. C. Huang, C. M. Feng, J. C. Shyu, "Noninvasive glucose monitoring in vivo with an optical heterodyne polarimeter," Appl. Opt. 37, 3553-3557 (1998).

3. I. A. Vitkin, E. Hoskinson, "Polarization studies in multiply scattering chiral media," Opt. Eng. 39, 353-362 (2000).

4. K. Hadley, I. Vikin, "Optical rotation and linear and circular depolarization rates in diffusively scattered light from chiral, racemic, and achiral turbid media," J. Biomed. Opt. 7, 291-299 (2002).

5. J. S. Maier, S. A. Walker, S. Fantini, M. A. Franceschini, E. Gratton, "Possible correlations between blood glucose concentration and the reduced scattering coefficient of tissues in the near infrared," Opt. Lett. 19, 2062-2064 (1994).

6. M. Kohl, M. Cope, M. Essenpreis, D. Bcker, "Influence of glucose concentration on light scattering in tissue-simulating phantoms," Opt. Lett. 19, 2170-2172 (1994).

7. J. T. Bruulsema, J. E. Hayward, T. J. Farrell, M. S. Patterson, L. Heinemann, M. Berger, T. Koschinsky, J. Sandahl-Christiansen, H. Orskov, M. Essenpreis, G. Schmelzeisen-Redeker, D. Böcker, "Correlation between blood glucose concentration in diabetics and noninvasively measured tissue optical scattering coefficient," Opt. Lett. 22, 190-192 (1997).
8. K. V. Larin, M. S. Eledrisi, M. Motamedi, R. O. Esenaliev, "Noninvasive blood glucose monitoring with optical coherence tomography: A pilot study in human subjects," Diabetes Care 25, 2263-2267 (2002).

9. R. O. Esenaliev, K. V. Larin, I. V. Larina, M. Motaedi, "Noninvasive monitoring of glucose concentration with optical coherence tomography," Opt. Lett. 26, 992-994 (2001).

10. Y. H. Chan, C. Chou, J. S. Wu, H. F. Chang, H. F. Yau, "Properties of a diffused photon-pair density wave in a multiple-scattering medium," Appl. Opt. 44, 1416-1425 (2005).

11. L. P. Yu, C. Chou, J. S. Wu, Y. H. Chan, "Measurement of diffuse photon-pairs density wave in a multiple-scattering medium," Appl. Opt. 47, 2708-2714 (2008).

12. I. Barman, C. R. Kong, G. P. Singh, R. R. Dasari, M. S. Feld, "Accurate spectroscopic calibration for noninvasive glucose monitoring by modeling the physiological glucose dynamics," Anal. Chem. 82, 6104-6114 (2010).

13. G. Purvinis, B. D. Camero, D. M. Altrogge, "Noninvasive Polarimetric-Based Glucose Monitoring: An in Vivo Study," J. Diabetes Sci. Technol. 5, 380-387 (2011).

14. T. Durduran, R. Choe, W. B. Baker, A. G. Yodh, "Diffuse optics for tissue monitoring and tomography," Rep. Prog. Phys. 73, 076701 (2010).

15. H. J. van Staveren, C. J. M. Moes, J. van Marie, S. A. Prahl, M. J. C. van Gemert, "Light scattering in intralipid- $10 \%$ in the wavelength range of $400-1100$ nm," Appl. Opt. 30, 4507-4514 (1991).

16. G. Jarry, E. Steimer, V. Damaschini, M. Epifanie, M. Jurczak, R. Kaiser "Coherence and polarization of light propagating through scattering media and biological tissues," Appl. Opt. 37, 7357-7367 (1998).

17. S. T. Flock, S. L. Jacques, B. C. Wilson, W. M. Star, M. J. C. van Gemert, "Optical properties of intralipid: A phantom medium for light propagation studies," Lasers Surg Med. 12, 510-519 (1992).

18. V. Thomsen, D. Schatzlein, D. Mercuro, "Limits of detection in spectroscopy," Spectroscopy 18, 112114 (2003).

19. C. M. Girardin, C. Huot, M. Gothier, E. Delvin, "Continuous glucose monitoring: A review of biochemical perspectives and clinical use in type 1 diabetes," Clin. Biochem. 42, 136-142 (2009).

20. R. C. Haskell, L. O. Svaasand, T. T. Tsay, T. C. Feng, M. S. McAdams, "Boundary conditions for the diffusion equation in radiative transfer," J. Opt. Soc. Am. A 11, 2727-2741 (1994).

21. X. D. Li, A. G. Yodh, B. Chance, D. N. Pattanayak, "Diffraction tomography for biochemical imaging with diffuse-photon density waves," Opt. Lett. 22, 573-575 (1997). 